\title{
Long Channel Carbon Nanotube as an Alternative to Nanoscale Silicon Channels in Scaled MOSFETs
}

\author{
Michael Loong Peng Tan \\ Faculty of Electrical Engineering, Universiti Teknologi Malaysia (UTM), 81310 Skudai, Johor, Malaysia \\ Correspondence should be addressed to Michael Loong Peng Tan; michael@fke.utm.my
}

Received 11 July 2013; Accepted 26 November 2013

Academic Editor: Chunyi Zhi

Copyright (C) 2013 Michael Loong Peng Tan. This is an open access article distributed under the Creative Commons Attribution License, which permits unrestricted use, distribution, and reproduction in any medium, provided the original work is properly cited.

Long channel carbon nanotube transistor (CNT) can be used to overcome the high electric field effects in nanoscale length silicon channel. When maximum electric field is reduced, the gate of a field-effect transistor (FET) is able to gain control of the channel at varying drain bias. The device performance of a zigzag CNTFET with the same unit area as a nanoscale silicon metaloxide semiconductor field-effect transistor (MOSFET) channel is assessed qualitatively. The drain characteristic of CNTFET and MOSFET device models as well as fabricated CNTFET device are explored over a wide range of drain and gate biases. The results obtained show that long channel nanotubes can significantly reduce the drain-induced barrier lowering (DIBL) effects in silicon MOSFET while sustaining the same unit area at higher current density.

\section{Introduction}

Carbon nanotubes (CNTs) are gaining momentum in the current silicon technology as a complementary nanostructure that could reform the device architecture. CNT modeling has been rigorously studied and examined [1-5] to assess the performance of the device at the circuit level. Advancement of the nanotechnology devices modeling is vital for the foreseeable future of carbon nanotube as switching device, interconnect and memory in integrated circuits (ICs). An in situ growth single-walled carbon nanotube (SWCNT), which integrates long channel $600 \mathrm{~nm}$ CNT channel, thin $\mathrm{Al}_{2} \mathrm{O}_{3}$ top gate contact, and Palladium (Pd) metal source/drain contacts, has been demonstrated [6].

In addition, we report the potential of long channel $65 \mathrm{~nm}$ CNT as substitute to $45 \mathrm{~nm}$ silicon metal-oxide semiconductor field-effect transistor (Si MOSFET) from the perspective of modeling for future CNT-logic applications. We observe good agreement between CNTFET and Si MOSFET respectively, when simulating two-terminal drain currentvoltage $\left(I_{d}-V_{d}\right)$ characteristic. The projection has shed light on the reduction of DIBL and high field effects [7] as well as reduction in long channel CNT which is a widespread phenomenon in nanoscale Si MOSFET $[8,9]$. We also demonstrate the effects of the channel area restructuring on the maximum electric field as well as density of states (DOS) in the conductance of CNT. Unlike MOSFET, it is revealed that the performance of CNT is enhanced when the source and drain width is minimized rather than the length, primarily due to the gate-to-source-drain parasitic fringe capacitances [10]. MOSFET scaling in accordance with Moore's Law will reach its fundamental limitation as a result of process controllability in the next 10 years. Consequently, it is necessary to ensure that novel material is studied to provide alternatives to the current technologies and challenges in the new era of nanotechnology.

\section{Carbon Nanotube and MOSFET Modeling}

The layout of a CNTFET device is depicted in Figure 1. The area of the channel is given by the multiplication of the width, $W$, of the source and drain contact and the length, $L$, of the nanotube [6]. Details of the quasiballistic MOSFET device modeling can be found in previous work in [11, 12].

The carbon nanotube model [13] is a unified nanostructure model based on quantum transport theory established by Datta [14]. This work extended the universal DOS spectral 


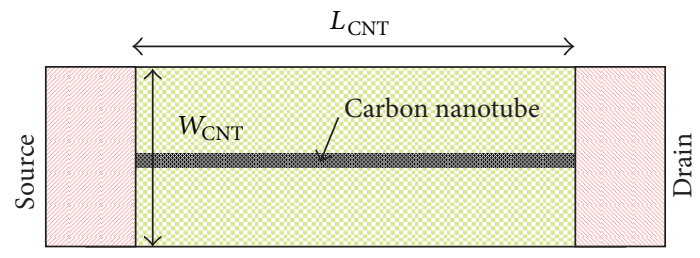

FIGURE 1: The unit area size of CNT channel with source and drain.

function [15] into the numerical calculation for CNT conduction subbands. We have included multiband density of states to account for multimode transport [16]. For an accurate simulation, the input parameters shown in Table 1 for MOSFET and CNTFET are extracted from TSMC [17] and Javey et al. [18], respectively. The $60 \mathrm{~nm}$ nanotube device model incorporate quasiballistic transport scattering as confirmed by [18]. At $60 \mathrm{~nm}$ length, the carriers travelling on the CNT surface have smaller mean free path than acoustic phonon which occurred at $300 \mathrm{~nm}$.

The typical width of a high-tech CNTFET device is reported [10] to be $1 \mu \mathrm{m}$. The width of the CNT is calculated to be $W_{\mathrm{CNT}}=A_{\mathrm{MOS}} / L_{\mathrm{C}}$, when both CNT and MOSFET devices are having identical channel area $\left(A_{\mathrm{MOS}}=A_{\mathrm{CNT}}\right)$. In a case when both devices can provide same level of current, channel area becomes $A=(k L)^{2}$ when given the scaling factor; $k$ and both parameter $W=L$. CNT channel with length, $2 k L_{\mathrm{CNT}}$, can provide the same current with $W_{\mathrm{CNT}}=0.5 L_{\mathrm{CNT}}$. Even when the physical widths of the CNT channel, $W \leq$ $0.5 k L_{\mathrm{CNT}}$, there is no area drawback provided $L \geq 2 k L_{\mathrm{CNT}}$. As nanotube channel length increases, maximum electric field in CNT, $E_{m \mathrm{CNT}}$ reduces tremendously $[19,20]$. As for CNT with $L=60 \mathrm{~nm}$, the maximum electric field is found to be $E_{m}=(3 / 4) E_{m \mathrm{Si}}$.

In the $I_{d}-V_{d}$ simulation of CNTFET, Landauer-Buttiker formalism is utilized [21]. The drain current, $I_{d}$ is given as

$$
\begin{aligned}
I_{d}\left(V_{G}, V_{d}, V_{s}\right) \\
=G_{\mathrm{ON}} \frac{k_{B} T}{q}\left[\log \left(1+\exp \left(q \frac{\left(E_{F}-V_{\mathrm{sc}}\left(V_{G}, V_{d}, V_{s}\right)\right)}{k_{B} T}\right)\right)\right] \\
\quad-G_{\mathrm{ON}} \frac{k_{B} T}{q} \\
\quad \times\left[\log \left(1+\exp \left(q \frac{\left(E_{F}-V_{\mathrm{sc}}\left(V_{G}, V_{d}, V_{s}\right)-V_{d}-V_{s}\right)}{k_{B} T}\right)\right)\right],
\end{aligned}
$$

where $G_{\mathrm{ON}}$ is the ON-conductance, $V_{\mathrm{sc}}$ is the channel potential, $E_{F}$ is the Fermi energy, $k_{B}$ is the Boltzmann Constant, $T$ is the temperature, $q$ is the charge of an electron, $V_{G}$ is the gate voltage, $V_{d}$ is the drain voltage, and $V_{s}$ is the source voltage.

The quantum conductance limit of a ballistic SWCNT is $G_{\mathrm{ON}}=4 q^{2} / h$. The theoretical framework of (1) derivation
TABLE 1: Device model input and output parameter at $V_{G}=1 \mathrm{~V}$.

\begin{tabular}{lcc}
\hline Parameter & CNT & MOSFET \\
\hline Type & Zigzag & NMOS \\
Structure & Q1D & Q2D \\
Gate insulator thickness, $t_{\text {ox }}$ & $1.1 \mathrm{~nm}$ & $1.1 \mathrm{~nm}$ \\
Channel length, $L$ & $60 \mathrm{~nm}$ & $45 \mathrm{~nm}$ \\
Channel width, $W$ & $67.5 \mathrm{~nm}$ & $90 \mathrm{~nm}$ \\
Channel area, $A$ & $4.05 \times 10^{-15} \mathrm{~m}^{2}$ & $4.05 \times 10^{-15} \mathrm{~m}^{2}$ \\
Tube diameter, $d$ & $1.5437 \mathrm{~nm}$ & - \\
Chiral vector $(n, m)$ & $(20,0)$ & - \\
Maximum electric field, $E_{m}$ & $0.75 E_{m S \mathrm{Si}}$ & $E_{m S \mathrm{Si}}$ \\
Maximum current, $I_{d \max }$ & $59.43 \mu \mathrm{A}$ & $65.2 \mu \mathrm{A}$ \\
Carrier density, $I_{d \max } /(d$ or $W)$ & $42.8 \mu \mathrm{A} / \mathrm{nm}$ & $0.8 \mu \mathrm{A} / \mathrm{nm}$ \\
DIBL & $39.41 \mathrm{mV} / \mathrm{V}$ & $56.23 \mathrm{mV} / \mathrm{V}$ \\
Subthreshold swing, SS & $74.44 \mathrm{mV} / \mathrm{dec}$ & $32.37 \mathrm{mV} / \mathrm{dec}$ \\
On-off ratio & $9.2 \times 10^{3}$ & $3.8 \times 10^{3}$ \\
\hline
\end{tabular}

can be found in $[19,20]$. The quasi-one-dimensional (Q1D) density of state function of CNT [22] is given by

$$
D(E)=\frac{2 g_{v} g_{s}}{3 \pi a_{c c} t} \sum_{i} \frac{E}{\sqrt{E^{2}-\left(E_{G} / 2\right)^{2}}},
$$

where $a_{\mathrm{cc}}=1.42 \AA$ and $t=3 \mathrm{eV}$ is the carbon-to-carbon $(\mathrm{C}-\mathrm{C})$ bonding energy, $E_{G}$ is the bandgap energy, $g_{s}$ is the spin degeneracy, and $g_{v}$ is the valley degeneracy. On the other hand, the $I_{d}-V_{d}$ characteristics for a short channel MOSFET can be expressed as

$$
\begin{array}{r}
I_{d}=\frac{C_{G} \mu_{\ell f} W_{\mathrm{MOS}}}{L} \frac{\left(\left(V_{G}-V_{T}\right)-(1 / 2) V_{d}\right) V_{d}}{1+V_{d} / V_{c}}, \\
0 \leq V_{d} \leq V_{d \text { sat }},
\end{array}
$$

where $C_{G}$ is the gate capacitance, $\mu_{\ell f}$ is the gate-field dependent mobility, $V_{d \text { sat }}$ is the saturation voltage at the point of current saturation, $V_{c}$ is the critical voltage, and $V_{T}$ is the threshold voltage. At current saturation, (3) becomes

$$
I_{d \text { sat }}=\alpha C_{G}\left(V_{G}-V_{T}-V_{d \text { sat }}\right) W v_{\text {sat }}, \quad V_{d} \geq V_{d \text { sat }},
$$

where $\alpha=v_{D} / v_{\text {sat }}$ is ratio of drift velocity, $v_{D}$ with saturation velocity and $v_{\text {sat }}$ at the drain $[11,23]$.

\section{Results and Discussion}

Figure 2 shows the density of states for Q1D of $[20,0]$ zigzag CNT with three van Hove singularities. As the energy span widens, more electrons are capable of occupying the singularities pinned between source and drain Fermi levels.

In the $I_{d}-V_{d}$ simulation, the source Fermi energy is set to be at $0.22 \mathrm{eV}$ below the conduction band. Our simulation results in Figure 3 which comes from (1) indicate that the CNT is able to offer drain current performance comparable to a $45 \mathrm{~nm}$ Si MOSFET. Remarkably, the effective current per 


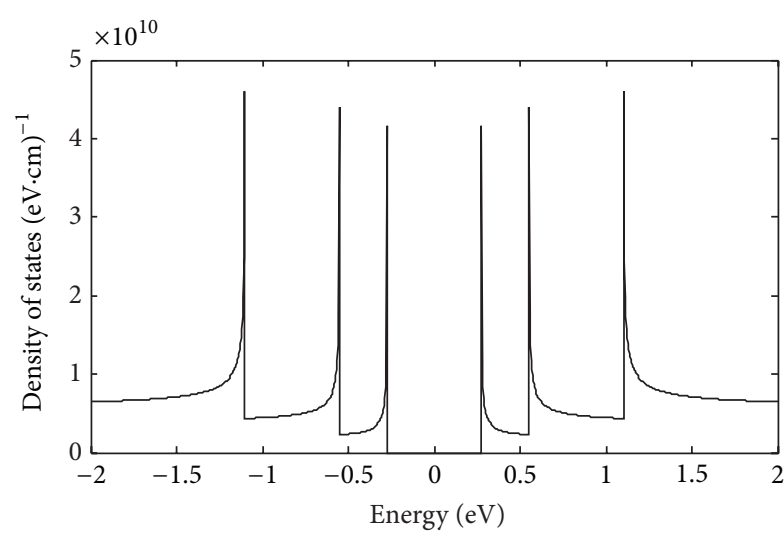

FIGURE 2: Electronic density of states calculated for [20,0] zigzag nanotubes.

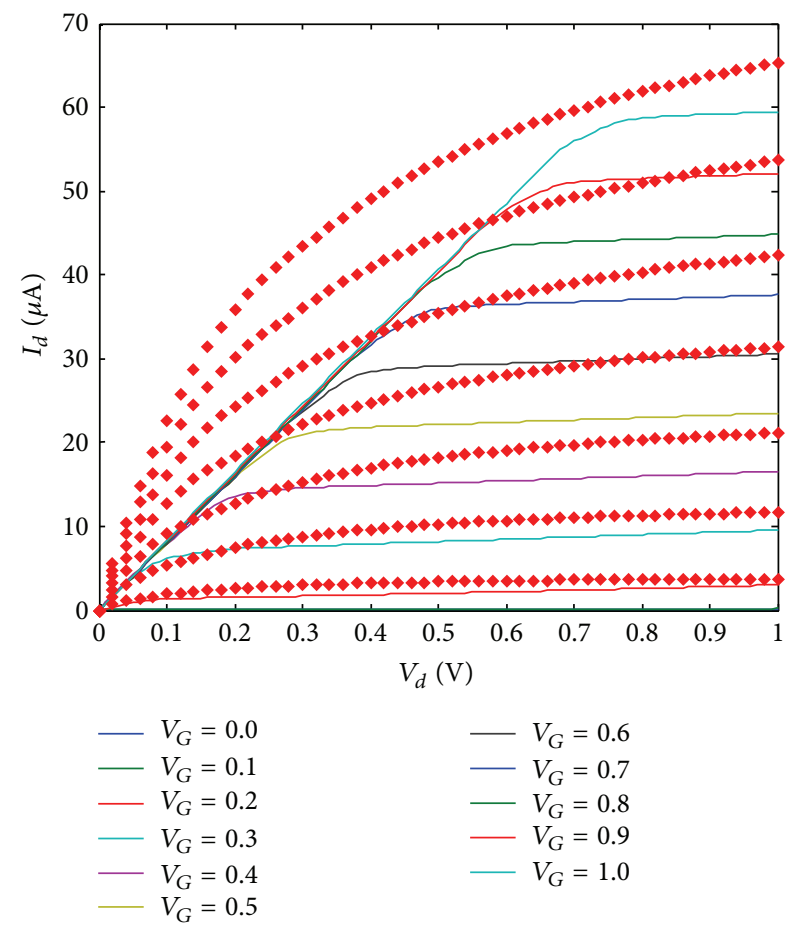

FIGURE 3: Drain characteristic of $60 \mathrm{~nm}$ single-walled carbon nanotube (colour solid lines) and $45 \mathrm{~nm}$ MOSFET (diamonds) with $0.1 \mathrm{~V}$ gate increment. Initial $V_{G}$ for MOSFET is $0.4 \mathrm{~V}$ (bottom).

unit dimension yielded 53.5 times more of Si channel because of the small diameter tube.

The DIBL effects is suppressed fairly well for both devices with a slight advantage to CNT. Silicon demonstrated a superior subthreshold swing at $32.37 \mathrm{mV} / \mathrm{dec}$, a value half of CNT. Although CNT has a lower on current, it sustains a high on-off ratio in 4 orders of magnitude. In addition to the device simulation, a SWCNT with a channel length of $600 \mathrm{~nm}$ is fabricated and shown in Figure 4. The Paladium contacts are made by electron beam lithography on SWCNT grown by thermal CVD from catalyst islands.

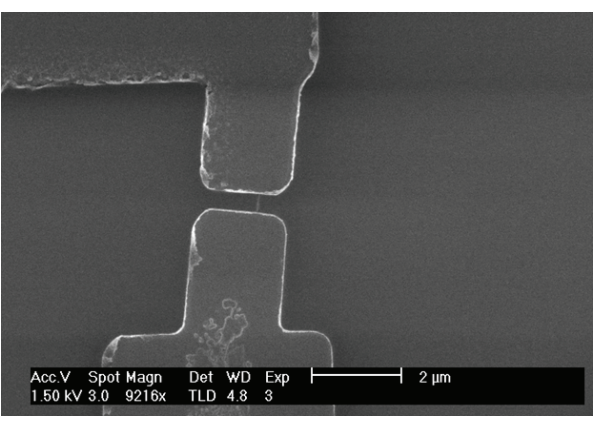

FIGURE 4: Plan view of SWCNT formed between Palladium source and drain contact.

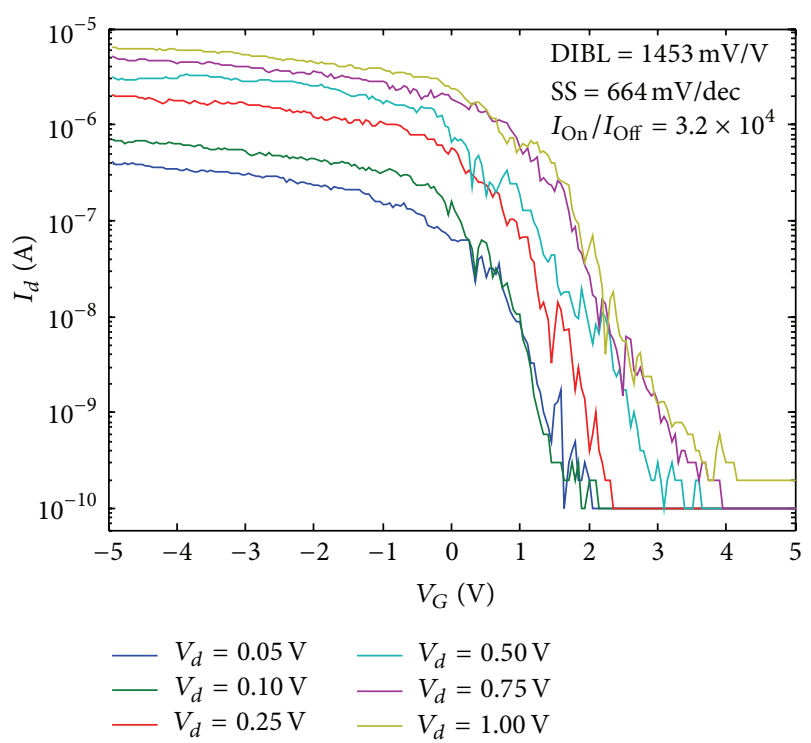

FIGURE 5: Gate characteristics measurement of a $600 \mathrm{~nm}$ CNT from $V_{d}=0.05$ to $V_{d}=1.0 \mathrm{~V}$.

The $I_{d}-V_{G}$ measurement was carried out on a back gate geometry $\approx 200 \mathrm{~nm} \mathrm{SiO}_{2}$ depicted in Figure 5. From Figure 3, the gate characteristic, $I_{d}-V_{G}$, can be generated for the $60 \mathrm{~nm}$ CNT model and it is illustrated in Figure 6. The DIBL for the experimental data is at $1453 \mathrm{mV} / \mathrm{V}$, while SS is estimated to be $664 \mathrm{mV} / \mathrm{dec}$. Nevertheless, the $600 \mathrm{~nm}$ fabricated CNT is compensated by a high off-on ratio at $3.2 \times 10^{4}$. It is found that DIBL can be lowered by at least one order of magnitude by doping the source end region of the channel [24] or the whole CNT $[25,26]$.

\section{Conclusion}

It is revealed that long channel CNT can deliver drain current comparable to a MOSFET. The carrier density along the CNTFET is at least 50 times that of the Si MOSFET. In the same channel area, CNT has better control of short channel effect (SCE) than $\mathrm{Si}$ as it has lower $E_{m}$. This brings an enormous advantage since lower $E_{m}$ has a smaller DIBL. A double gate or a cylindrical gate structure has the best control 


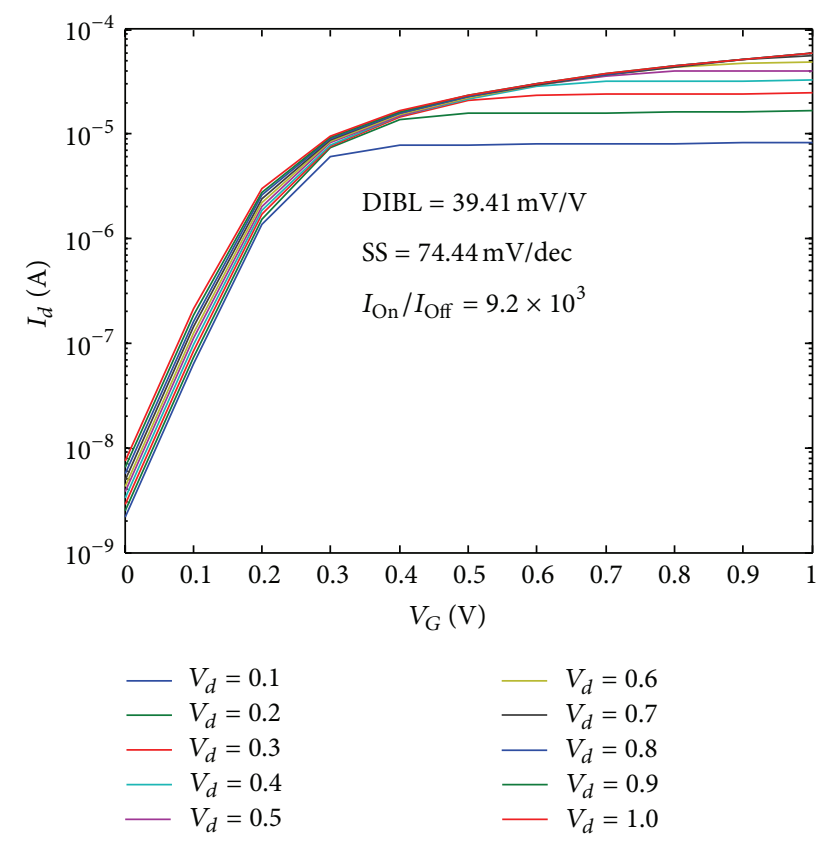

FIGURE 6: Gate characteristics of a $60 \mathrm{~nm}$ CNT model from $V_{d}=0.1$ to $V_{d}=1.0 \mathrm{~V}$ in $0.1 \mathrm{~V}$ increment.

to suppress DIBL [27]. Based on this, we could have lower offcurrent in the transistor. As a result, a CNT uses less power consumption as a switching device when operating at the same frequency as a MOSFET.

\section{Acknowledgments}

MLPT thanks the Ministry of Higher Education Malaysia and the Universiti Teknologi Malaysia (UTM) for the award of advanced study fellowship leading to a Ph.D. degree at the University of Cambridge. This work is partially supported by UTM Research University Grant vot no. Q.J130000.2623.09J21 and Fundamental Research Grant Scheme (FRGS/2/2013/SG02/UTM/02/2). MLPT is also immensely grateful to Aun Shih Teh for providing the experimental data of SWCNT. The author also thanks the Research Management Centre (RMC) of UTM and Centre for Advanced Photonics and Electronics (CAPE), Cambridge, UK, for providing an excellent research environment to complete this work.

\section{References}

[1] J. Deng and H.-S. P. Wong, "A compact SPICE model for carbonnanotube field-effect transistors including nonidealities and its application. Part I: model of the intrinsic channel region," IEEE Transactions on Electron Devices, vol. 54, no. 12, pp. 3186-3194, 2007.

[2] J. Deng and H.-S. P. Wong, "A compact SPICE model for carbonnanotube field-effect transistors including nonidealities and its application. Part II: full device model and circuit performance benchmarking," IEEE Transactions on Electron Devices, vol. 54, no. 12, pp. 3195-3205, 2007.
[3] J. Guo, S. Datta, M. Lundstrom et al., "Assessment of silicon MOS and carbon nanotube FET performance limits using a general theory of ballistic transistors," in Proceedings of the IEEE International Devices Meeting (IEDM), pp. 711-714, December 2002.

[4] J. M. Marulanda, A. Srivastava, and A. K. Sharma, "Transfer characteristics and high frequency modeling of logic gates using carbon nanotube field effect transistors (CNT-FETs)," in Proceedings of the 20th Symposium on Integrated Circuits and System Design, pp. 202-206, Rio de Janeiro, Brazil, September 2007.

[5] K. Natori, Y. Kimura, and T. Shimizu, "Characteristics of a carbon nanotube field-effect transistor analyzed as a ballistic nanowire field-effect transistor," Journal of Applied Physics, vol. 97, no. 3, Article ID 034306, 2005.

[6] G. A. J. Amaratunga, A. S. Teh, S. N. Cha et al., "Nanotube and nanowire transistors," in Proceedings of the Interational Semiconductor Conference (CAS '05), vol. 1, pp. 3-8, October 2005.

[7] V. K. Arora and M. L. P. Tan, "High-field transport in graphene and carbon nanotubes," in Proceedings of the IEEE International Conference of Electron Devices and Solid-State Circuits (EDSSC '13), pp. 1-2, 2013.

[8] M. T. Ahmadi, R. Ismail, M. L. P. Tan, and V. K. Arora, "The ultimate ballistic drift velocity in carbon nanotubes," Journal of Nanomaterials, vol. 2008, Article ID 769250, 8 pages, 2008.

[9] R. Vidhi, M. L. P. Tan, T. Saxena, A. M. Hashim, and V. K. Arora, "The drift response to a high-electric-field in carbon nanotubes," Current Nanoscience, vol. 6, no. 5, pp. 492-495, 2010.

[10] B. C. Paul, S. Fujita, M. Okajima, and T. Lee, "Modeling and analysis of circuit performance of ballistic CNFET," in Proceedings of the 43rd Design Automation Conference, pp. 717-722, 2006.

[11] V. K. Arora, M. L. P. Tan, I. Saad, and R. Ismail, "Ballistic quantum transport in a nanoscale metal-oxide-semiconductor field effect transistor," Applied Physics Letters, vol. 91, no. 10, Article ID 103510, 2007.

[12] M. L. P. Tan, R. Ismail, R. Muniandy, and V. K. Wong, "Velocity saturation dependence on temperature, substrate doping concentration and longitudinal electric field in nanoscale MOSFET," in Proceedings of the IEEE National Symposium on Microelectronics, 2005.

[13] A. Rahman, J. Guo, S. Datta, and M. S. Lundstrom, "Theory of ballistic nanotransistors," IEEE Transactions on Electron Devices, vol. 50, no. 9, pp. 1853-1864, 2003.

[14] S. Datta, Electronic Transport in Mesoscopic Systems, Cambridge University Press, Cmabridge, UK, 1994.

[15] J. W. Mintmire and C. T. White, "Universal density of states for carbon nanotubes," Physical Review Letters, vol. 81, no. 12, pp. 2506-2509, 1998.

[16] J. Appenzeller, J. Knoch, M. Radosavljević, and P. Avouris, "Multimode transport in schottky-barrier carbon-nanotube field-effect transistors," Physical Review Letters, vol. 92, no. 22, Article ID 226802, 2004.

[17] Z. Dawei, Z. Hao, Y. Zhiping, and T. Lilin, "A unified charge model comprising both $2 \mathrm{D}$ quantum mechanical effects in channels and in poly-silicon gates of MOSFETs," Solid-State Electronics, vol. 49, no. 10, pp. 1581-1588, 2005.

[18] A. Javey, J. Guo, M. Paulsson et al., "High-field quasiballistic transport in short carbon nanotubes," Physical Review Letters, vol. 92, no. 10, Article ID 106804, 2004. 
[19] M. L. P. Tan and G. A. J. Amaratunga, "Performance prediction of graphene nanoribbon and carbon nanotube transistors," in Proceedings of the AIP Conference Proceedings,, vol. 1341, pp. 365-369, 2011.

[20] M. L. P. Tan, G. Lentaris, and G. A. Amaratunga, "Device and circuit-level performance of carbon nanotube field-effect transistor with benchmarking against a nano-MOSFET," Nanoscale Research Letters, vol. 7, article 467, 2012.

[21] Y. Imry and R. Landauer, "Conductance viewed as transmission," Reviews of Modern Physics, vol. 71, no. 2, pp. S306-S312, 1999.

[22] D. C. Y. Chek, M. L. P. Tan, M. T. Ahmadi, R. Ismail, and V. K. Arora, "Analytical modeling of high performance singlewalled carbon nanotube field-effect-transistor," Microelectronics Journal, vol. 41, no. 9, pp. 579-584, 2010.

[23] M. L. P. Tan, V. K. Arora, I. Saad, M. Taghi Ahmadi, and R. Ismail, "The drain velocity overshoot in an $80 \mathrm{~nm}$ metaloxide-semiconductor field-effect transistor," Journal of Applied Physics, vol. 105, no. 7, Article ID 074503, 2009.

[24] Z. Arefinia and A. A. Orouji, "Novel attributes in the performance and scaling effects of carbon nanotube field-effect transistors with halo doping," Superlattices and Microstructures, vol. 45, no. 6, pp. 535-546, 2009.

[25] J. Chen, C. Klinke, A. Afzali, and P. Avouris, "Self-aligned carbon nanotube transistors with charge transfer doping," Applied Physics Letters, vol. 86, no. 12, Article ID 123108, pp. 1-3, 2005.

[26] K. Alam and R. Lake, "Role of doping in carbon nanotube transistors with source/drain underlaps," IEEE Transactions on Nanotechnology, vol. 6, no. 6, pp. 652-659, 2007.

[27] N. Bahador, M. L. P. Tan, M. T. Ahmadi, and R. Ismail, "A unified drain-current model of silicon nanowire field-effect transistor (SiNWFET) for performance metric evaluation," Science of Advanced Material, vol. 6, pp. 354-360, 2014. 

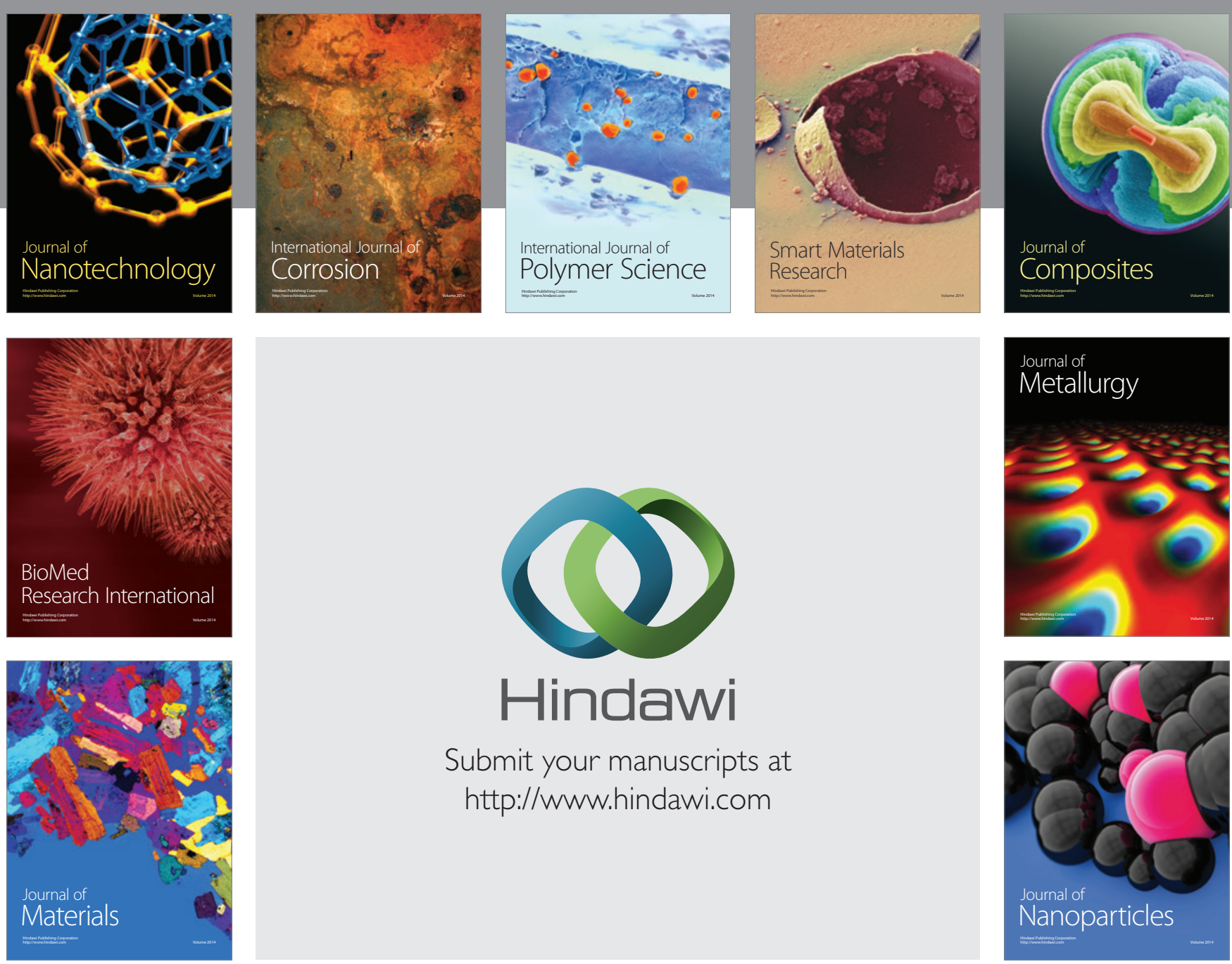

Submit your manuscripts at http://www.hindawi.com
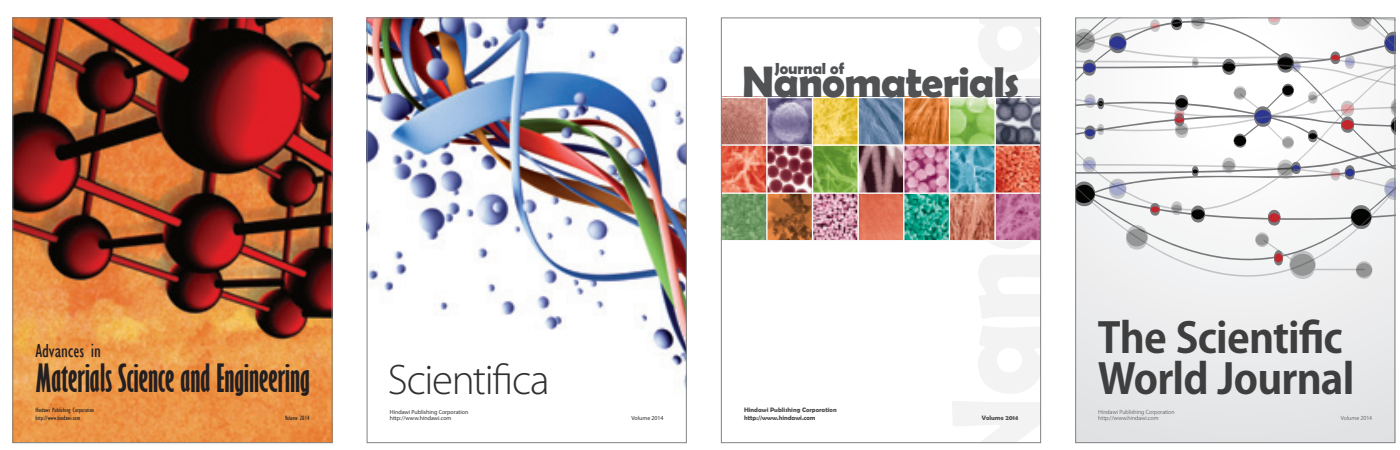

\section{The Scientific World Journal}
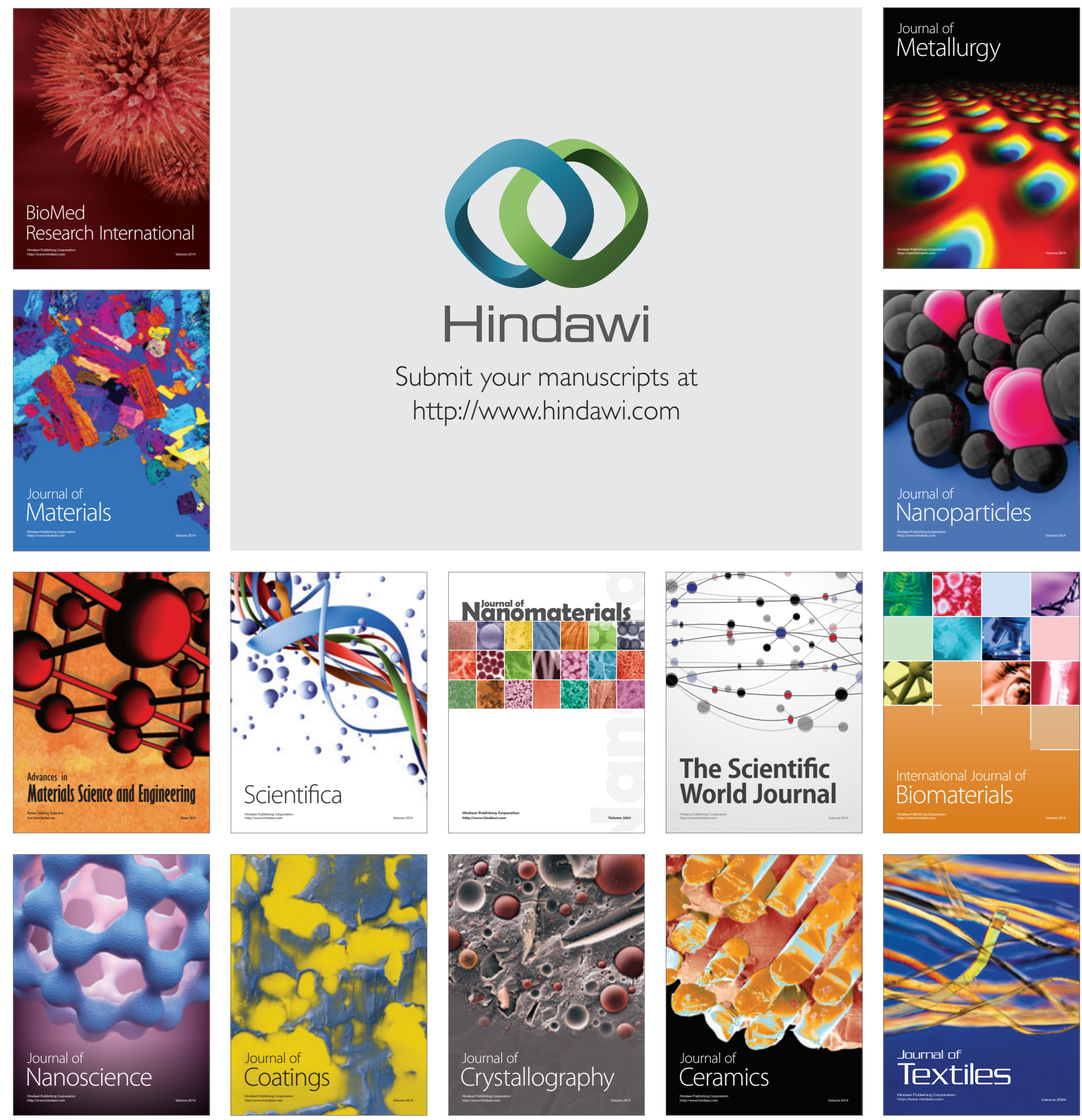